\title{
Urinary cGMP concentrations after exposure to radio-contrast media in patient at risk for contrast media induced nephropathy (CIN) predicts 90-day morbidity and mortality
}

\author{
Berthold Hocher ${ }^{1,2^{*}}$, Fabian Heunisch ${ }^{1,2}$, Gina-Franziska von Einem ${ }^{1,2}$, Markus Alter ${ }^{1,2}$, Yvonne Keim³, \\ Johannes-Peter Stasch ${ }^{3}$
}

From 6th International Conference on cGMP: Generators, Effectors and Therapeutic Implications Erfurt, Germany. 28-30 June 2013

\section{Background}

Acute renal failure after exposure to contrast media in patients at risk for CIN increases substantially 90-day mortality and morbidity. Biomarkers for outcome after CIN are missing so far.

\section{Results}

We analyzed in a prospective study 233 patients with either diabetes or preexisting impaired kidney function getting intra-arterial contrast media (CM) for coronary angiograms. Urinary cGMP concentrations were analyzed 24 hours after exposure to intra-arterial CM in spot urine. The patients were followed for 90 day for the following events: death, need for transient or permanent dialysis, doubling of serum creatinine and unplanned re-hospitalization. 51 out of the 233 patients had such an event during the follow up period of 90 days after contrast media exposure. The urinary cGMP to creatinine ratio was significantly increased in patients having an event during the follow up period ( $\mathrm{p}=0.011$ ). We furthermore performed a multivariate regression analysis considering age, baseline kidney function before contrast media exposure and preexisting diabetes - all important risk factors for CIN. These analysis revealed that the urinary cGMP to creatinine ratio is an in dependent predictor of the occurrence of the composite endpoint: death, need for transient or permanent dialysis, doubling of serum creatinine and unplanned re-hospitalization $(\mathrm{p}=0.010 ; \mathrm{B}=0.001 ; \mathrm{T}=2.584$; $\mathrm{CI}=0.001-0.003)$.

\section{Conclusion}

The urinary cGMP to creatinineratio isan independent predictor of major advers events after contrast media exposure during a follow-up of 90 days.

\section{Authors' details}

'Institute for Nutritional Science, University of Potsdam, Potsdam, Germany. ${ }^{2}$ Center for Cardiovascular Research, Charité, Berlin, Germany. ${ }^{3}$ Bayer Pharma AG, Wuppertal, Germany

Published: 29 August 2013

\section{doi:10.1186/2050-6511-14-S1-P29}

Cite this article as: Hocher et al:: Urinary cGMP concentrations after exposure to radio-contrast media in patient at risk for contrast media induced nephropathy (CIN) predicts 90-day morbidity and mortality. BMC Pharmacology and Toxicology 2013 14(Suppl 1):P29.

*Correspondence: berthold.hocher@charite.de

1 Institute for Nutritional Science, University of Potsdam, Potsdam, Germany

Full list of author information is available at the end of the article

C 2013 Hocher et al; licensee BioMed Central Ltd. This is an Open Access article distributed under the terms of the Creative Commons 